\title{
Spatially Resolved Raman Spectroscopy of Single- and Few-Layer Graphene
}

\author{
Davy Graf * Françoise Molitor, and Klaus Ensslin \\ Solid State Physics Laboratory, ETH Zurich, 8093 Zurich, Switzerland \\ Christoph Stampfer, Alain Jungen, and Christofer Hierold \\ Micro and Nanosystems, ETH Zurich, 8092 Zurich, Switzerland \\ Ludger Wirtz \\ Institute for Electronics, Microelectronics, and Nanotechnology (IEMN), \\ CNRS-UMR 8520, B.P. 60069, 59652 Villeneuve d'Ascq Cedex, France
}

(Dated: February 4, 2008)

\begin{abstract}
We present Raman spectroscopy measurements on single- and few-layer graphene flakes. Using a scanning confocal approach we collect spectral data with spatial resolution, which allows us to directly compare Raman images with scanning force micrographs. Single-layer graphene can be distinguished from double- and few-layer by the width of the D' line: the single peak for single-layer graphene splits into different peaks for the double-layer. These findings are explained using the double-resonant Raman model based on ab-initio calculations of the electronic structure and of the phonon dispersion. We investigate the $\mathrm{D}$ line intensity and find no defects within the flake. A finite $\mathrm{D}$ line response originating from the edges can be attributed either to defects or to the breakdown of translational symmetry.
\end{abstract}

PACS numbers: Valid PACS appear here

Keywords: Raman mapping

The interest in graphite has been revived in the last two decades with the advent of fullerenes 1 and carbon nanotubes ${ }^{2}$ However, only recently single- and few-layer graphene could be transferred to a substrate ${ }^{\frac{3}{\underline{m}}}$ Transport measurements revealed a highly-tunable two-dimensional electron/hole gas of relativistic Dirac Fermions embedded in a solid-state environment ${ }^{4.5}$ Going to few-layer graphene, however, disturbs this unique system in such a way that the usual parabolic energy dispersion is recovered. The large structural anisotropy makes few-layer graphene therefore a promising candidate to study the rich physics at the crossover from bulk to purely twodimensional systems. Turning on the weak interlayer coupling while stacking a second layer onto a graphene sheet leads to a branching of the electronic bands and the phonon dispersion at the $\mathrm{K}$ point. Double-resonant Raman scattering ${ }^{6}$ which depends on electronic and vibrational properties turns out to be an ingenious tool to probe the lifting of that specific degeneracy.

We report on Raman mapping of single- and few-layer graphene flakes resting on a silicon oxide substrate. Lateral resolution of $400 \mathrm{~nm}$ allows to address neighboring sections with various layers of graphene down to a single graphene sheet, previously determined with the scanning force microscope (SFM). We find that the integrated $\mathrm{G}$ line signal is directly correlated with the thickness of the graphitic flake and is shifted upward in frequency for double- and single-layer graphene compared to bulk graphite. The mapping of the peak width of the D' line shows a strong contrast between single- and few-layer graphene. Such a pronounced sensitivity to the transition to the very last layer offers an optical and nondestructive method to unambiguously detect single-layer graphene. In addition, we locally resolve the structural quality of the flake by investigating the $\mathrm{D}$ band, which is related to elastic backscattering. The map of the integrated D line signal of a graphitic flake with doubleand single-layer sections shows that the inner part of the flake is quasi defect free, whereas edges and steps serves as scatterers. Finally, we explain the splitting of the D' line as a function of the number of graphene layers within the double-resonant Raman model ${ }^{6}$ The comparison between experimental data and theory confirms the qualitative validity of the double-resonant Raman model, but demonstrates quantitative differences between theory and experiment. In particular, the model, when based on first-principles calculations, predicts a much smaller splitting of the peaks.

The graphite films were prepared by mechanical exfoliation of highly oriented pyrolytic graphite (HOPG) and subsequent transfer to a highly doped $\mathrm{Si}$ waver with $300 \mathrm{~nm} \mathrm{SiO}_{2}$ (atomic oxidation process) cap layer ${ }^{3.7}$ The combination of optical microscopy using phase contrast and SFM makes it possible to locate flakes with various thicknesses down to a monolayer with lateral extensions in the micrometer range. The Raman spectra were acquired using a laser excitation of $532 \mathrm{~nm}(2.33 \mathrm{eV})$ delivered through a single-mode optical fiber, whose spot size is limited by diffraction. Using a long working distance focusing lens with a numerical aperture $\mathrm{NA}=0.80$ we obtain a spot size of about $400 \mathrm{~nm}$. With a very low incident power of 4-7 $\mu \mathrm{W}$ heating effects can be neglected.

The Raman spectrum of graphite has four prominent peaks (Fig. [3- for a recent review see Ref. 8). The peak around $1582 \mathrm{~cm}^{-1}$, commonly called G line, is caused by the Raman active $E_{2 g}$ phonon, (in-plane optical mode) 


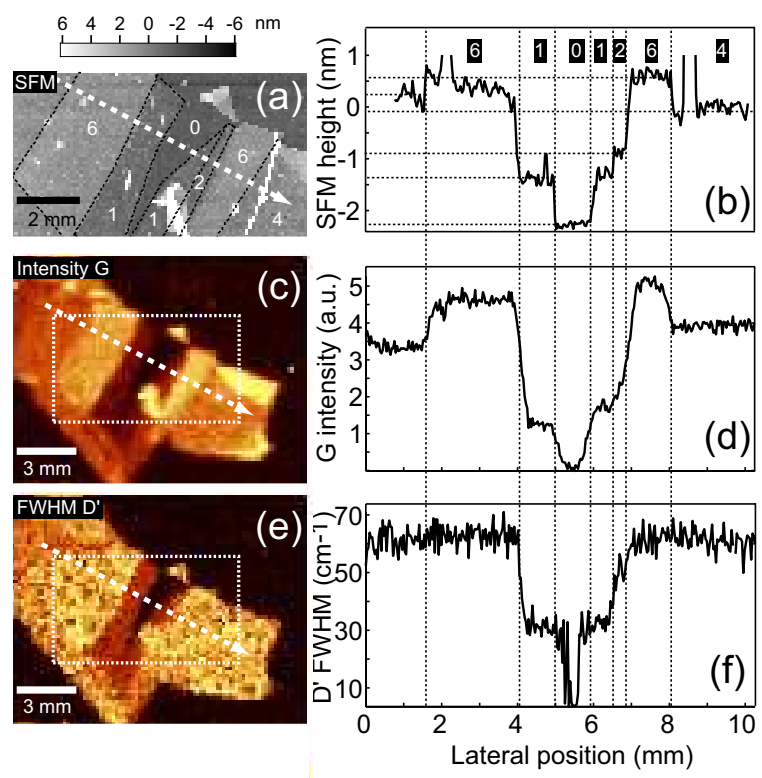

FIG. 1: (a),(b) SFM micrograph and cross-sectional plot (indicated with the white dashed arrow; lateral average over 400 $\mathrm{nm}$ ) of a few-layer graphene flake with central sections down to a single layer. Raman maps (dashed square corresponding to the SFM image in (a)) showing (c) the integrated intensity of the G line and (e) the FWHM of the D' line. The related cross sections (d),(f) are aligned (vertical dashed lines) with the height trace.

close to the $\Gamma$ point. The $\mathrm{D}$ line around $1350 \mathrm{~cm}^{-1}$ exhibits two remarkable features: its position shifts to higher frequencies with increasing incident laser excitation energies ${ }^{9}$ and its relative signal strength (compared to the G line) depends strongly on the amount of disorder in the graphitic material ${ }^{9.10}$ The associated overtone D' around $2700 \mathrm{~cm}^{-1}$ is pronounced even in the absence of a D signal. Finally, the overtone of the G line, the G' line, is located at $3248 \mathrm{~cm}^{-1}$, which is more than twice the energy of the $\mathrm{G}$ line. The different experimental findings related to the dispersive $\mathrm{D}$, D' bands could be explained by Thomsen and Reich within the framework of double resonant Raman scattering,,$\frac{6}{,}$ which was extended to other phonon branches by Saito et al: $\stackrel{11}{n}$ The electronic and vibrational properties of graphite are dominated by the $\mathrm{sp}^{2}$-nature of the strong intraplane covalent bonds. The relatively weak inter-layer coupling causes the high structural anisotropy. Raman spectra for multiple graphene layers can be compared qualitatively and quantitatively while investigating flakes with sections of various thicknesses. In Fig. 1(a) the SFM micrograph of a graphite flake with different layers is presented: The bare $\mathrm{SiO}_{2}$ (indicated by ' 0 ') is surrounded by single-layer sections with steps of up to two, six and four layers. The different step heights are clearly depicted in Fig. T(b), where a cross section of Fig. 1(a) (see white dashed arrow) is shown. Scanning the flake and collecting for each spot the com-
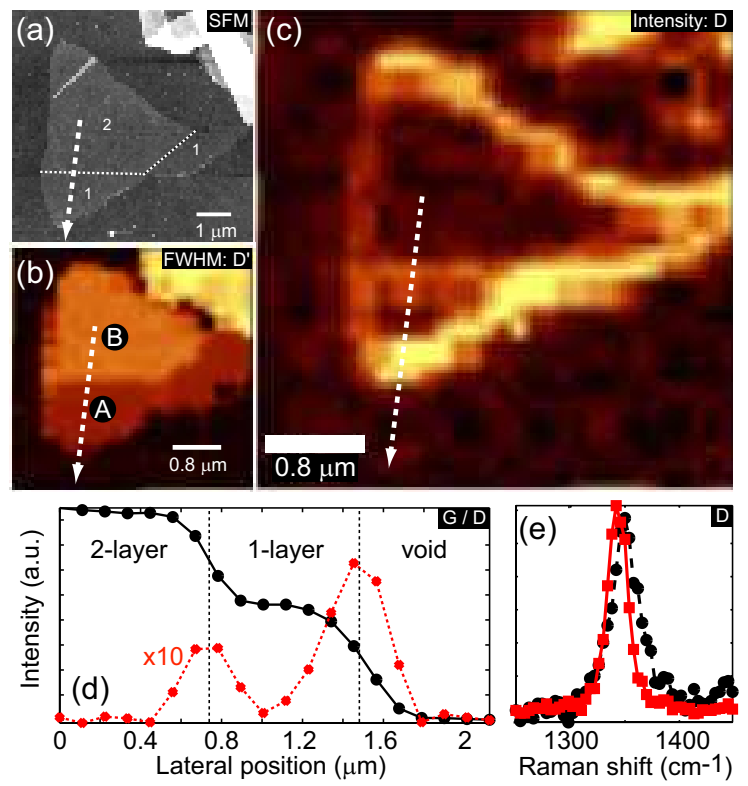

FIG. 2: (a) SFM micrograph of a graphitic flake consisting of one double- and two single-layer sections (white dashed line along the boundaries), highlighted in the Raman map (b) showing the FWHM of the D' line. (c) Raman mapping of the integrated intensity of the D line: A strong signal is detected along the edge of the flake and at the steps from double- to single-layer sections. (d) Raman cross section (white dashed arrow in (c)): Staircase behavior of the integrated intensity of the $\mathrm{G}$ peak (solid line) and pronounced peaks at the steps for the integrated intensity of the D line (dashed line). (e) Spatially averaged D peak for the crossover from double to single layer $\left(\bullet\right.$, dashed line) and from single layer to the $\mathrm{SiO}_{2}$ substrate (ם, solid line).

plete Raman spectrum we can subsequently filter specific spectral data for spatially resolved data point and construct false-color 2D plots. In Fig. I(c) the intensity of the $\mathrm{G}$ peak is integrated from 1537 to $1622 \mathrm{~cm}^{-1}$. We find a remarkable correlation with the SFM graph: Brighter regions correspond to thicker sections. The cross section in Fig. 1(d) shows a step-like behavior, perfectly correlated with the topographical changes shown in Fig. 1(b). In Fig. I(e) we plot the FWHM (full width at half maximum) of the D' line. It shows the narrowing at the transition to a single-layer (see e.g. Fig. 31) and gives an evident contrast between single- and few-layer graphene sections. The quasi digital change from about 60 to 30

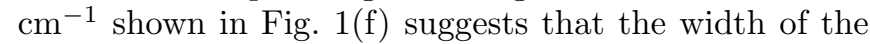
D' line can be used as a detector for single-layer graphene resting on a substrate. Raman spectroscopy can therefore be used to count the layers of a thin graphite stack and to discriminate between single and double layer. Combined with the double-resonant Raman scattering mechanism an optical setup using light in the visible range turns out to be an alternative to scanning force microscopy, which requires stacking folds as height references.

Transport measurements show that the quality of the 


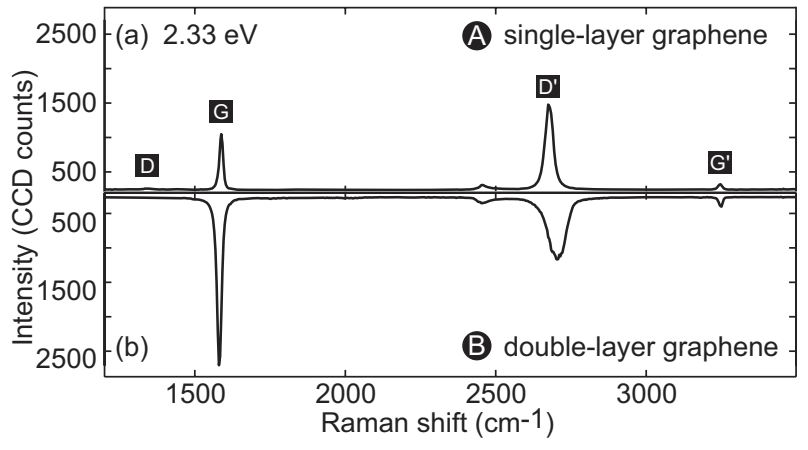

FIG. 3: Raman spectra of (a) single- and (b) double-layer graphene (collected at spots A and B, see Fig. 2(b)).

finite graphitic flakes on the silicon oxide matrix obtained with the technique explained above is remarkable: electronic mobilities up to $15^{\prime} 000 \mathrm{~cm}^{2}(\mathrm{Vs})^{-1}$ were estimated

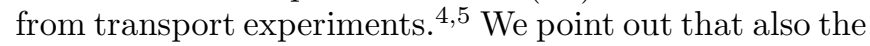
Raman spectroscopy reveals quasi defect free graphitic sheets via the absence of a D band signal. First experiments have related the intensity of the $\mathrm{D}$ band to the structural coherence of the graphite material. In fact it is inversely proportional to the crystallite grain size $\underline{10}$ The appearance of the $\mathrm{D}$ band can, however, be related to the occurrence of defects and disorder in general, as shown in experiments with boron-doped and electrochemically oxidized HOPG 12 With micro-Raman mapping we are able to localize the spatial origin of the defects. From crosscorrelating the SFM micrograph in Fig. 2(a) with the Raman map of the integrated D line $\left(1300-1383 \mathrm{~cm}^{-1}\right)$ intensity in Fig. 2(c) we infer directly that the edges of the flake and also the borderline between sections of different height contribute to the D band signal whereas the inner parts of the flakes do not. This is somewhat surprising since for thinner flakes the influence of a nearby substrate on the structural quality should be increasingly important. In the cross-section Fig. 22(d) we see clearly that the $\mathrm{D}$ line intensity is maximal at the section boundaries, which can be assigned to translational symmetry breaking or to defects. However, we want to emphasize that the D line is still one order of magnitude smaller than the G line. In Fig. 2(e) spatially averaged D mode spectra from the two steps shown in Fig. 2(d) are presented. The frequency fits well into the linear dispersion relation of peak shift and excitation energy found in earlier experiments $\frac{9}{} \mathrm{In}$ addition, we find that the peak is narrower and down-shifted at the edge of the single-layer while it is somewhat broader and displays a shoulder at the crossover from the double to the single layer.

In Fig. 3(a) and (b), we compare the Raman spectra of the double- and single-layer graphene shown in Fig. 2(b) and labeled with A and B. The Raman signal is significantly altered when peeling off the penultimate layer: the $G$ peak decreases strongly in intensity and shifts towards higher wave numbers. In connection with Fig. I (b) we already stated that the integrated G line
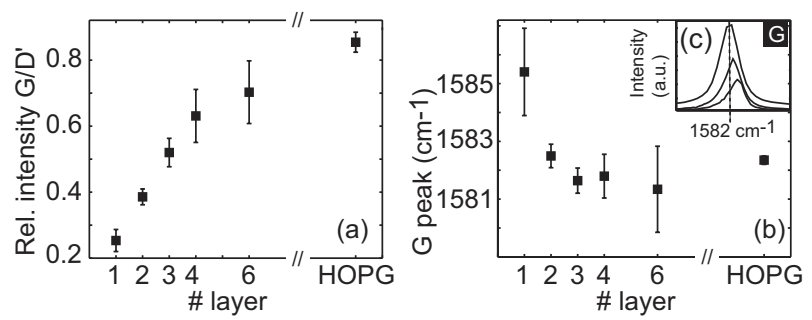

FIG. 4: (a) Plot of the ratio of the integrated intensities of the $G$ and D' peak versus number of stacked layers (average value and standard deviation). (b) G line frequency versus number of stacked layers (average value and standard deviation). (c) G peak for HOPG (upper peak), double- (middle peak) and single-layer (lower peak) graphene. The vertical dashed line indicates the reference value for bulk graphite.

signal is monotonously increasing with increasing flake thickness. In order to compare data of different flakes and measurement runs we turn our attention to the ratio of the integrated intensities of the $\mathrm{G}$ and $\mathrm{D}$ ' line, plotted in Fig. 廿(a). Most of the changes can be attributed to the decrease of the $G$ line, since the spectral weight of the D' band changes only slightly. The intensity ratio increases almost linearly from one to four layers. In Fig. 4 (b) the dependence of the $G$ peak position on the layer number is investigated. Spectral data of various sections on different flakes were averaged. The frequency shifts towards higher wave numbers at the crossover to the double- and especially to the single-layer graphene. However, in the case of single-layer graphene, it is accompanied by an important statistical spread of the collected data. In Fig. [4(c) representative $\mathrm{G}$ peak spectra for single-, doublelayer graphene and HOPG are presented. It is important to note that in contrast to the $\mathrm{G}$ line, the corresponding overtone band, the G' line, does not change its spectral position as a function of the number of layers.

The most prominent difference in the spectra of singlelayer, few-layer, and bulk graphite lies in the D' line: the integrated intensity of the D' line stays almost constant, even though it narrows to a single peak at lower wave number at the crossover to a single layer (Fig. [3). The width of the D' peak or - at high resolution - its splitting into different sub-peaks (Fig. [5) is in the following explained in the framework of the double-resonant Raman model $\underline{\underline{6}}$ The model explains the D' line in the following way (see Fig. 6(a)): An electron is vertically excited from point $\mathrm{A}$ in the $\pi$ band to point $\mathrm{B}$ in the $\pi^{*}$ band by absorbing a photon. The excited electron is inelastically scattered to point $\mathrm{C}$ by emission of a phonon with momentum $q$. Since the energy of this phonon $(\approx 150 \mathrm{meV})$ is small compared with the photon energy of $2.33 \mathrm{eV}$, we have drawn the line horizontally, for simplicity. Inelastic backscattering to the vicinity of point A by emission of another phonon with momentum $\approx q$ and electron-hole recombination lead to emission of a photon with an energy about $300 \mathrm{meV}$ less than the energy of the incident photon. In principle, two other double-resonant Raman 


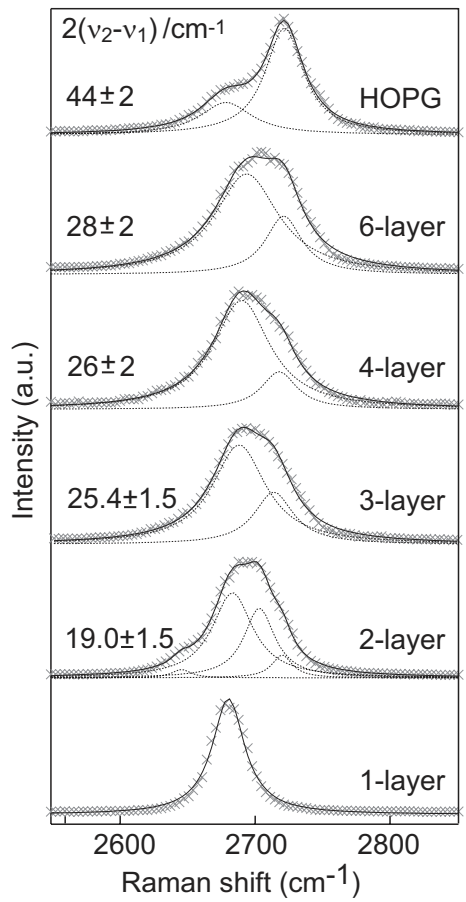

FIG. 5: D' peaks for an increasing number of graphene layers along with HOPG as a bulk reference. The dashed lines show the Lorentzian peaks used to fit the data, the solid lines are the fitted results. The single peak position for the singlelayer graphene is at $2678.8 \pm 1.0 \mathrm{~cm}^{-1}$. The peak position of the the two inner most peaks for double-layer graphene are $2683.0 \pm 1.5$ and $2701.8 \pm 1.0 \mathrm{~cm}^{-1}$. On the left the value for the splitting from double-layer graphene up to HOPG is presented. All peaks are normalized in amplitude and vertically offset.

processes, involving the phonons $q^{\prime}$ and $q^{\prime \prime}$, are possible as well. However, it was argued in Ref. 13 that their weight is very low: 14

In Fig. [6] we compare the electronic band structure of the single layer with the ones of the double layer and of bulk graphite. All three dispersion relations were calculated from first-principles, using density-functional theory in the local density approximation $\stackrel{15}{\underline{15}}$ Ine doublelayer, the $\pi$ and $\pi^{*}$ bands split into two bands each. This gives rise to four different possible excitations. We have calculated the corresponding oscillator strengths 16 and found that for the excitation energy of $2.33 \mathrm{eV}$, transitions $1-3$ and $2-4$ have negligible weight, while transitions 1-4 and 2-3 (displayed in Fig. 6(b)) have almost equal weight. For each of the two dominant vertical transitions, there are two possible horizontal transitions. The corresponding electron-phonon coupling matrix elements for the phonons $q_{0}$ to $q_{3}$ are almost equal ${ }^{17}$ In theory, we therefore expect a splitting of the D' band into four peaks of almost equal height. Our experimental data (Fig. 515) shows indeed that the D' line for the double layer can be decomposed into four peaks. However, the outer two peaks (corresponding to the phonons $q_{0}$ and $q_{3}$ ) have very low weight in the experimental data. We calculated
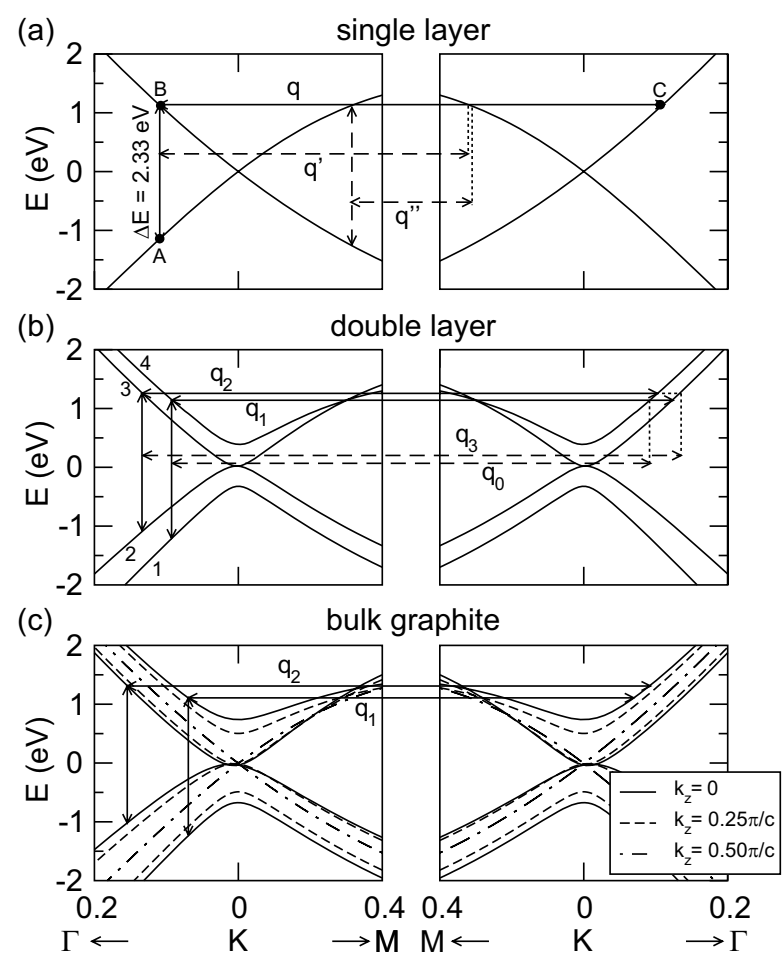

FIG. 6: Electronic band structure along the high-symmetry lines $\Gamma-\mathrm{K}$ and K-M: (a) single-layer graphene, (b) double-layer graphene, and (c) bulk graphite. For bulk graphite, we display the band structure in the direction parallel to the graphene planes for different values of the transverse momentum $k_{z}$. Vertical arrows denote vertical transitions by $2.33 \mathrm{eV}$ from a valence $(\pi)$ band to a conductance $\left(\pi^{*}\right)$ band. Horizontal arrows denote transitions between two states of almost equal energy by coupling to a phonon of momentum $q_{i}$ (the corresponding phonon frequencies are displayed in Table (). Dashed horizontal lines denote transitions with considerably less weight than the solid horizontal lines (see text).

from first principles $\frac{15}{5}$ the phonon-frequencies $\nu_{1}$ and $\nu_{2}$, corresponding to the momenta $q_{1}$ and $q_{2}$. The frequencies of the highest optical branch are given in Table — Due to the weak inter-layer coupling the degeneracy of this branch is lifted. However, the frequency difference remains weak $\left(<1 \mathrm{~cm}^{-1}\right)$ and does not significantly contribute to the experimentally observed splitting about 19 $\mathrm{cm}^{-1}$ of the D' line (see Fig. [5). Table】furthermore gives the value for $2\left(\nu_{2}-\nu_{1}\right)$. We note that the value obtained from our first-principles calculation is only half as large as the experimentally observed splitting of about $19 \mathrm{~cm}^{-1}$. This discrepancy is related to the fact, that the doubleresonant Raman model based on ab-initio calculations also predicts a value for the dispersion of the D' line with incident laser energy that amounts only to about half of the experimentally observed value of $99 \mathrm{~cm}^{-1} / \mathrm{eV}$ : 18 We conclude therefore that the double-resonant Raman model can qualitatively explain the fourfold splitting of the D' line in the double-layer, but the amount of the splitting and the relative heights of the peaks are not 


\begin{tabular}{|l|c|c||c|}
\hline & $\nu_{1} / \mathrm{cm}^{-1}$ & $\nu_{2} / \mathrm{cm}^{-1}$ & $2\left(\nu_{2}-\nu_{1}\right) / \mathrm{cm}^{-1}$ \\
\hline \hline bulk & $1393.2 / 1393.6$ & $1402.9 / 1403.1$ & $19.4 / 19.0$ \\
\hline double layer & $1395.6 / 1395.6$ & $1400.0 / 1400.6$ & $8.8 / 10.0$ \\
\hline single layer & \multicolumn{2}{|c||}{1398.1} & - \\
\hline
\end{tabular}

TABLE I: Frequencies of the optical phonons involved in the double-resonant Raman model. The corresponding phonon momenta $q_{1}$ and $q_{2}$ are determined from the ab-initio electronic band structures of Fig. [6] The splitting of the frequencies in the double-layer and bulk is due to the (weak) inter-layer interaction.

properly described within this model $\frac{19}{}$

In bulk graphite, the $\pi$ and $\pi^{*}$ bands split into a continuum of bands, i.e., they disperse in the direction $k_{z}$ perpendicular to the layer. In Fig. 6(c), we display the bands for three different values of $k_{z}$. In the joint-density of states, the vertical transitions for $k_{z}=0$ have the dominant weight and are thus considered in our calculations. Since the splitting between the bands is much more pronounced than for the double-layer, the value for $2\left(\nu_{2}-\nu_{1}\right)$ is about a factor of two higher than for the double layer. This is in agreement with the experimental data, see Fig. [5 where the splitting increases likewise by about a factor of two between the double layer and bulk graphite. As in the case of the double layer, there are quantitative differences between theory and experiment for graphite as well: First-principles calculations of the oscillator strengths and of electron-phonon coupling matrix elements predict an almost equal height of the peaks whereas the experiment shows that the lowerfrequency peak has a strongly reduced weight. The peaks corresponding to the horizontal transitions $q_{0}$ and $q_{3}$ are missing altogether in the experimental spectrum.

Even though some quantitative differences remain, the double-resonant Raman model explains well the observed differences in the D' line as we go from the single-layer via few-layer systems to the bulk limit. The quantitative differences may be an indicator that some essential effects are not properly included in the model. E.g., the role of quasi-particle effects (electron-electron interaction) ${ }^{20}$ and of excitonic effects (electron-hole interaction) in the double-resonance process remains to be understood. The importance of these effects has been recently demonstrated for electronic excitations in carbon nanotubes

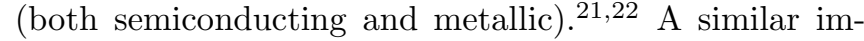
portance may be therefore expected for processes that involve electronic excitations in graphite ${ }^{23}$

In conclusion, Raman mapping reveals to be a powerful tool to investigate single- and few-layer graphene flakes. It turns out that the width of the D' line is highly sensitive to the crossover from single- to double-layer graphene, which is explained by a peak splitting following the double-resonant Raman model together with $a b$ initio electronic band structure calculations. A remaining open question is the decrease of the $\mathrm{G}$ line intensity with decreasing layer number compared to the almost constant spectral weight of the D' line and the accompanied upshift of its frequency for double- and single-layer graphene. The structural quality of the flakes is studied by analyzing the $\mathrm{D}$ line intensity: no defects are detected in the inner part of the flake. The D line signal from the boundaries of the individual sections of the flake suggest that they act as elastic scatterer.

The authors are grateful to Hubert Heersche for useful advices on sample preparation. We acknowledge stimulating discussions with A. Rubio and M. Lazzeri. Calculations were performed at IDRIS (project 061827). Financial support from the Swiss Science Foundation (Schweizerischer Nationalfonds) is gratefully acknowledged. L. W. acknowledges support from the French National Research Agency.
* Electronic address: grafdavy@phys.ethz.ch

1 H.W. Kroto, J.R. Heath, S.C. Obrien, R.F. Curl, R.E. Smalley, Nature 318, 162 (1985)

2 S. Iijima, Nature 354, 56 (1991)

3 K.S. Novoselov, A.K. Geim, S.V. Morozov, D. Jiang,Y. Zhang, S.V. Dubonos, I.V. Grigorieva, A.A. Firsov, Science 306, 666 (2004)

${ }^{4}$ K.S. Novoselov, A.K. Geim, S.V. Morozov, D. Jiang, M.I. Katsnelson, I.V. Grigorieva, S.V. Dubonos, A.A. Firsov, Nature 438, 197 (2005)

5 Yuanbo Zhang, Yan-Wen Tan, Horst L. Stormer, Philip Kim, Nature 438201 (2005)

${ }^{6}$ C. Thomsen and S. Reich, Phys. Rev. Lett. 85, 5214 (2000)

7 K. S. Novoselov, D. Jiang, F. Schedin, T. J. Booth, V. V. Khotkevich, S. V. Morozov, and A. K. Geim, PNAS 102, 10451 (2005)

8 S. Reich and C. Thomsen, Phil. Trans. R. Soc. Lond. A 362, 2271 (2004)

9 R. P. Vidano, D.B. Fischbach, L.J. Willis, T.M. Loehr, Solid State Commun. 39, 341 (1981)
10 F. Tuinstra and J.L. Koenig, J. Chem. Phys. 53, 1126 (1970)

11 R. Saito, A. Jorio, A. G. Souza Filho, G. Dresselhaus, M. S. Dresselhaus, and M. A. Pimenta, Phys. Rev. Lett. 88, 027401 (2002)

12 Y. Wang, D.C. Alsmeyer, and R.L. McCreery, Chem. Mater. 2, 557 (1990)

13 A.C. Ferrari, J.C. Meyer, V. Scardaci, C. Casiraghi, M. Lazzeri, F. Mauri, S. Piscanec, Da Jiang, K. S. Novoselov, S. Roth, and A. K. Geim, cond-mat/0606284 (2006)

14 The process involving $q^{\prime}$ (a phonon very close to the Kpoint) does not occur because the corresponding electronphonon coupling matrix element is approximately 0 (see footnote [24] of Ref. 24). And the process involving $q^{\prime \prime}$ has a low weight because due to the "trigonal warping" effect, the corresponding phase space volume in the twodimensional Brillouin zone is small compared to the phase space volume for process involving $q$.

15 We use the code ABINIT:X. Gonze et al., Comp. Mat. Sci. 25, 478 (2002). 
16 We use the computer code SELF written by A. Marini (http://www.fisica.uniroma2.it/ self/).

17 M. Lazzeri, private communication.

18 We note that Ferrari et al ${ }^{13}$ overcome this difficulty by using the semiempirical parameter $d \omega / d q$ (caption of Table I of Ref. 13) which is fitted to the experimental dispersion of the D' peak. We have avoided the use of this semiempirical parameter in order to assess the quantitative validity of the double-resonant Raman model based on first-principles calculations. The good agreement between experimental and theoretical D peak dispersion in Ref. 6 is based on a corresponding fit of the hopping parameter in the tight-binding band-structure.

19 For an exact quantitative evaluation of the peak heights, an integration in the two-dimensional Brillouin zone for all transitions of energy $\Delta E=2.33 \mathrm{eV}$ would have to be performed.

20 For a calculation of the quasiparticle bandstructure of graphite in the GW approximation see: S.G. Louie, in Top- ics in Computational Materials in Science, edited by C.Y. Fong (World Scientific, Singapore, 1997), p.96.

${ }^{21}$ C. D. Spataru, S. Ismail-Beigi, L. X. Benedict, and S. G. Louie, Phys. Rev. Lett. 92, 077402 (2004).

22 E. Chang, G. Bussi, A. Ruini, and E. Molinari, Phys. Rev. Lett. 92, 196401 (2004).

23 We note that the picture of independent electron-hole pair excitations has been quantitatively very successful for the assignment of nanotube spectra to the chiral indices $(m, n)$. However, this success was based on the use of tight-binding band-structures with correspondingly fitted parameters. A parameter-free, first-principles, calculation of the optical spectra of carbon nanotubes must take into account electron-electron and electron-hole interaction, and we suggest that this may be true for graphite as well.

24 S. Piscanec, M. Lazzeri, F. Mauri, A.C. Ferrari, and J. Robertson, Phys. Rev. Lett. 93, 185503 (2004). 